\title{
REVIEW \\ Evaluation of Anti-inflammatory and Anti-allergic Effects of Food Components Using DNA Microarray Analysis
}

\author{
Masuko KOBORI ${ }^{{ }^{*}}$, Kanji NAGAI ${ }^{1,2}$, Yumiko TAKAHASHI ${ }^{1}$ and Hideaki OIKE ${ }^{1}$ \\ 1 National Food Research Institute, National Agriculture and Food Research Organization \\ (Tsukuba, Ibaraki 305-8642, Japan) \\ 2 Mitsubishi Rayon Co., Ltd. (Yokohama, Kanagawa 230-0053, Japan)
}

\begin{abstract}
Many people suffer from allergies, such as asthma, pollinosis and atopic dermatitis, as well as inflammatory diseases. To elucidate the anti-allergic and anti-inflammatory effects of food components, we developed a DNA microarray carrying genes related to immunity, inflammation and housekeeping. Results of gene expression analysis using the DNA microarray showed good reproducibility and correlated well with those of quantitative RT-PCR analysis. DNA microarray analysis showed that the butanol soluble fraction of bitter gourd placenta extract suppressed bacterial lipopolysaccharide (LPS)-induced inflammatory gene expression in mouse macrophage-like cells (RAW264.7). DNA microarray analysis demonstrated that the flavonoid fisetin suppressed the activated $\mathrm{T}$ cell membrane induction of gene expression in HMC-1 human mast cells. Thus, we demonstrated the anti-inflammatory effect of bitter gourd and anti-allergic effect of fisetin using DNA microarray analysis. Furthermore, DNA microarray analysis detected gene expression associated with skin irritation using a reconstructed human epidermal model.
\end{abstract}

Discipline: Food

Additional key words: allergy, bitter gourd, fisetin, inflammation, mast cells

\section{Introduction}

The number of allergy and rheumatoid arthritis sufferers has increased to over $30 \%$ of the population of Japan. The diet is a promising factor that could alleviate these diseases. Therefore, we attempted to develop a tool that efficiently evaluates the anti-allergic and antiinflammatory functionality of diet.

During allergic and inflammatory responses, the expression of many immune and inflammatory genes drastically increases in tissues. DNA microarray is a chip carrying an arrayed series of DNA oligonucleotide sequences. DNA microarray, carrying probes to hybridize a specific gene target, can detect changes in gene expression levels. To evaluate the allergic and inflammatory conditions of tissues and the suppressive effects of food components, we developed a DNA microarray carrying genes related to immunity, inflammation and housekeeping ((Allergy Chip) Genopal ${ }^{\mathrm{TM}}$, Mitsubishi Rayon Co.).

\section{Suppressive effect of bitter gourd extract on inflammatory responses ${ }^{7}$}

Bacterial lipopolysaccharide (LPS) induces inflammatory responses in RAW264.7 mouse macrophage-like cells. LPS induces the inflammatory gene expression and secretion of pro-inflammatory cytokines, such as TNF $\alpha$, IL1 $\beta$ and IL18. Therefore, we first determined the LPS-induced expression of TNF $\alpha$ and other inflammatory genes using the DNA microarray we developed. Figure 1 shows the procedure for determining inflammatory gene expression in untreated and LPS-treated RAW264.7 cells. Each gene expression level is detected as fluorescence intensity by a DNA microarray reader (Biochip reader MB-M2C Yokogawa Electric Co.). LPS induced expression of 65 of the 204 genes carried on the mouse DNA microarray. Using the total RNA sample of LPS-treated or untreated RAW264.7 cells, we then determined the data reproducibility and quantitative

*Corresponding author: e-mail kobori@affrc.go.jp

Received 10 August 2009; accepted 30 October 2009. 
1.

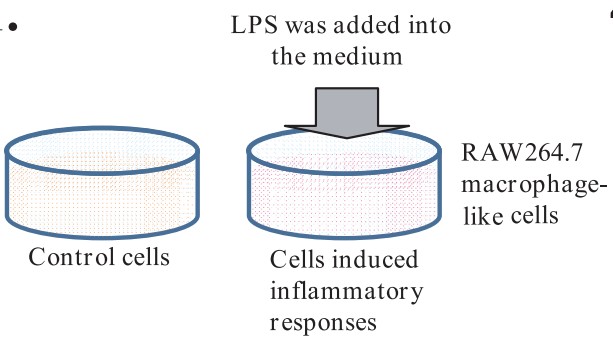

2.

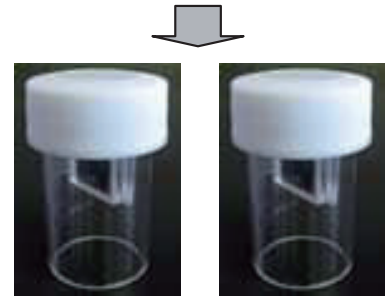

Total RNA of the cells was pretreated and hybridized to DNA microarray

3.

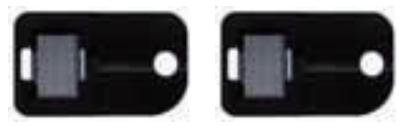

4.

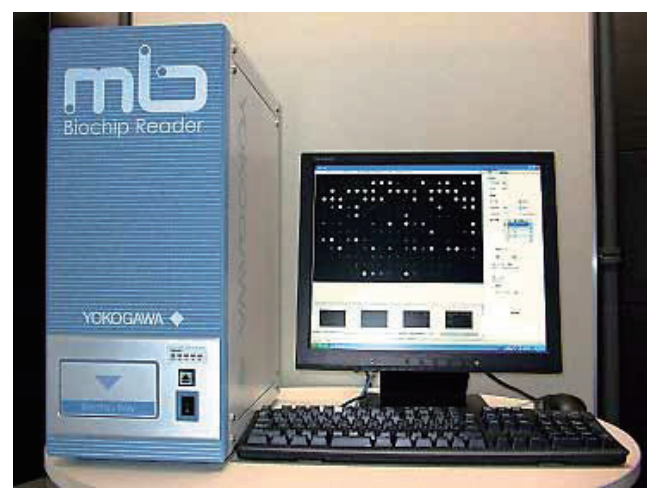

Gene expression levels were quantified by fluor escence intensity

5.

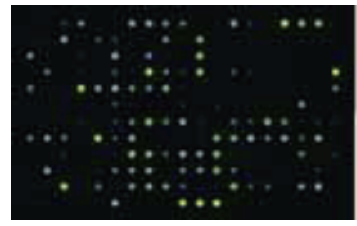

Untreated control cells

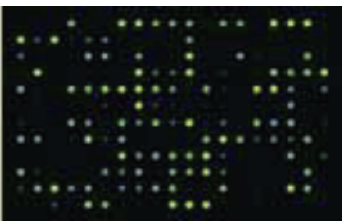

Cells induced the inflammatory responses by LPS

Fig. 1. DNA microarray detection of inflammatory gene expression in RAW264.7 cells induced by lipopolysaccharide (LPS)

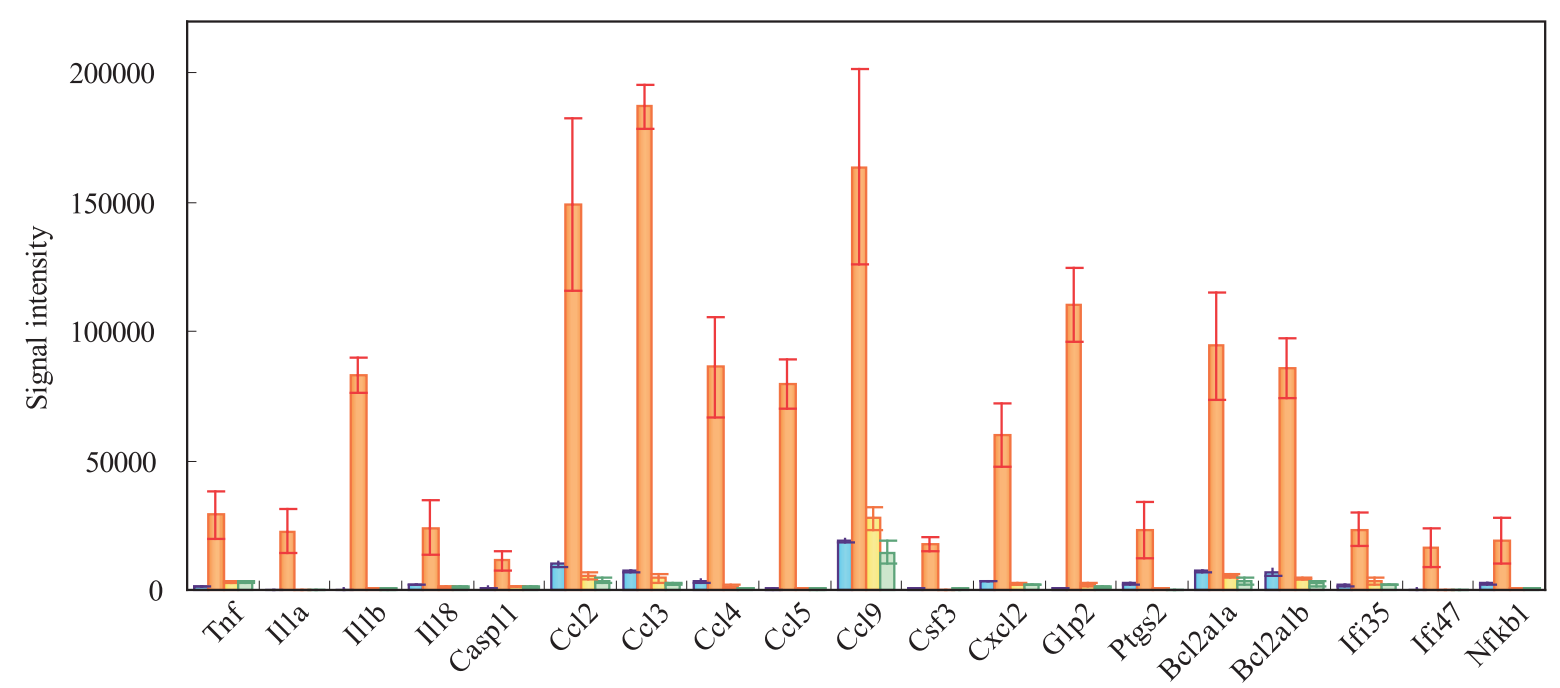

Fig. 2. Bitter gourd butanol fraction (Bitter gourd) suppresses LPS-induced inflammatory gene expression in RAW264.7 cells

Cells $\left(1 \times 10^{5}\right.$ cells $\left./ \mathrm{ml}\right)$ were treated with LPS $(2 \mathrm{ng} / \mathrm{ml})$ and the bitter gourd butanol fraction (bitter gourd) for $6 \mathrm{~h}$. Inflammatory gene expression was determined using a mouse DNA microarray (Genopal ARIM-GX, Mitsubishi Rayon Co., Ltd.). Data are expressed as mean \pm standard error from three different experiments.

$\square$ : control, $\square$ : LPS, $\square$ : Bitter gourd $50 \mu \mathrm{g} / \mathrm{ml}, \quad \square$ : Bitter gourd $100 \mu \mathrm{g} / \mathrm{ml}$. 
capacity of the DNA microarray analysis. As a result, the correlation coefficient between the fluorescence intensity data of two DNA chips applied with an equal amount of the same sample was 0.99 . The correlation coefficient between the results of the DNA microarray analysis and quantitative reverse transcription (RT)-PCR was 0.93 . The DNA microarray analysis showed good quantitative performance and provided highly reproducible results.

Bitter gourd (Momordica charantia L.) is a common vegetable in tropical areas of Asia and Africa. It has been traditionally used as a bitter stomachic and an anti-diabetic therapy. In our study on food functionality, we found that the butanol soluble fraction of bitter gourd placenta extract suppressed LPS-induced gene expression to the control level ${ }^{7}$ (Fig. 2). LPS induces inflammatory gene expression through the induction of $\mathrm{NF} \kappa \mathrm{B}$ and $\mathrm{C} / \mathrm{EBP}$ transcriptional activities, and MAPKs activities. We examined the effect of the bitter gourd butanol fraction on LPS-induced NFKB and C/EBP $\beta$ DNA binding activities in RAW264.7 cells. The NF- $\mathrm{KB}$ and $\mathrm{C}$ /EBP promoter binding activity in the nuclear extract were determined by monitoring their affinity to an immobilized oligonucleotide containing NF- $\mathrm{KB}$ and $\mathrm{C} / \mathrm{EBP}$ consensus binding site, respectively, and detected by ELISA. The butanol fraction significantly suppressed the DNA binding activity of NFKB but not C/EBP $\beta$. Furthermore, the bitter gourd butanol fraction suppressed the activation of MAPKs p38, JNK, and ERK. Our results suggest that the bitter gourd butanol fraction suppressed LPS-induced inflammatory gene expression by inhibiting NFKB DNA binding and following transcriptional activities, and the activation of MAPKs in RAW 264.7 cells. We identified the active components which suppressed LPS-induced inflammatory gene expression in the bitter gourd butanol fraction as 1- $\alpha$-linolenoyl lysophosphatidylcholine (LPC), 2- $\alpha$-linolenoyl LPC, 1lynoleoyl LPC, and 2-linoleoyl LPC.

Since bitter gourd was suggested to suppress LPSinduced inflammation, we determined the effect of bitter gourd juice on LPS and anti-collagen antibody-induced arthritis in Balb/c mice. As a result of daily oral administration of bitter gourd juice, the thickness of the inflamed and swollen hind feet was significantly reduced after 8 days. Oral administration of control (3\% glucose) significantly reduced the thickness of hind feet only after 12 days. Our result suggests that bitter gourd juice improved LPS- and anti-collagen antibody-induced arthritis in Balb/c mice.

\section{Suppressive effect of the flavonoid fisetin on activated $\mathrm{T}$ cell-induced activation of $\mathrm{HMC}-1$ mast cells ${ }^{8}$}

Mast cells play an important role in allergies such as asthma and pollinosis. Mast cells are activated by IgE-cross linking, release chemical mediators such as histamines, prostaglandins, cytokines, and chemokines, and develop the symptoms of allergy. In addition, mast cells are activated by cell-to-cell interaction with $\mathrm{T}$ cells, and this is thought to induce cutaneous delayed-type hypersensitivity (a $\mathrm{T}$ cell-mediated allergic inflammatory response), airway inflammation and remodeling of asthma. To evaluate the activation of HMC-1 human mast cells induced by activated $\mathrm{T}$ cell membranes, we determined the gene expression induced by activated $\mathrm{T}$ cell membranes in HMC-1 cells using DNA microarray. The DNA microarray analysis showed that activated $\mathrm{T}$ cell membranes induced the expression of 34 genes, including those for chemokines, interleukins and cell surface membrane proteins, of the 205 genes carried on the human DNA microarray (Fig. 3).

Fisetin (3,7,3',4'-tetrahydroxyflavone) (Fig. 4) is a flavonoid contained in vegetables and fruits such as onions, strawberries and apples ${ }^{1}$. Fisetin was shown to suppress the degranulation and IL4 and IL13 production in RBL-2H3 rat basophilic cells stimulated by IgE-cross linking ${ }^{5,6,10}$. We first examined the effect of fisetin on IgE/antigen-stimulated degranulation of LAD2 human mast cells. Fisetin suppressed IgE cross-linkingmediated activation of LAD2 cells. We then examined the effect of fisetin on activated $\mathrm{T}$ cell-induced activation of HMC-1 cells. Activated T cell membranes induced cell spreading and adhesion of HMC-1 cells and fisetin suppressed the morphological changes of the cells (Fig. 5). Furthermore, fisetin significantly suppressed gene expression induced by activated $\mathrm{T}$ cell membranes in HMC-1 cells (Fig. 3). Fisetin suppressed the secretion of granzyme B (GZMB) and perfolin (PRF), as well as their gene expression. Fisetin was suggested to suppress gene expression in HMC-1 cells stimulated by activated $\mathrm{T}$ cell membranes by inhibiting NFKB transcriptional activity.

Drosera rotundifolia, containing flavonoids, naphthoquinones, ellagic acid, and other polyphenols, has been used in the therapy of respiratory tract infections as the traditional medicine Drosera Herba. We found that the OASIS HLB column-absorbed fractions of $D$. rotundifolia and Drosera tokaiensis, which was suggested to be an amphidiploid between $D$. rotundifolia and Drosera spatulata Labill., suppressed the activated $\mathrm{T}$ cell membrane-induced gene expression in HMC-1 


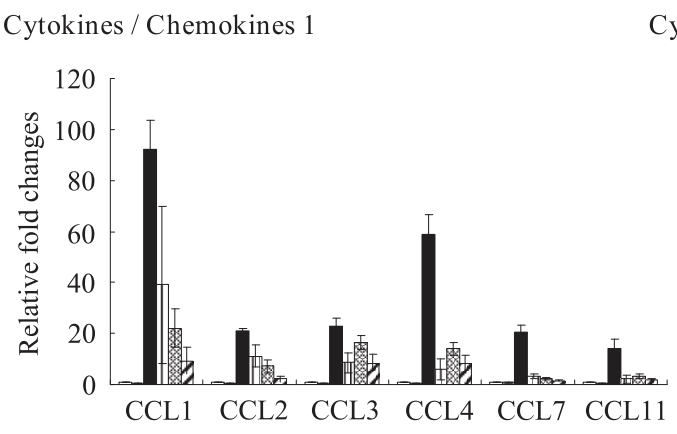

Cytokines / Chemokines 2

Cell surface membranes proteins

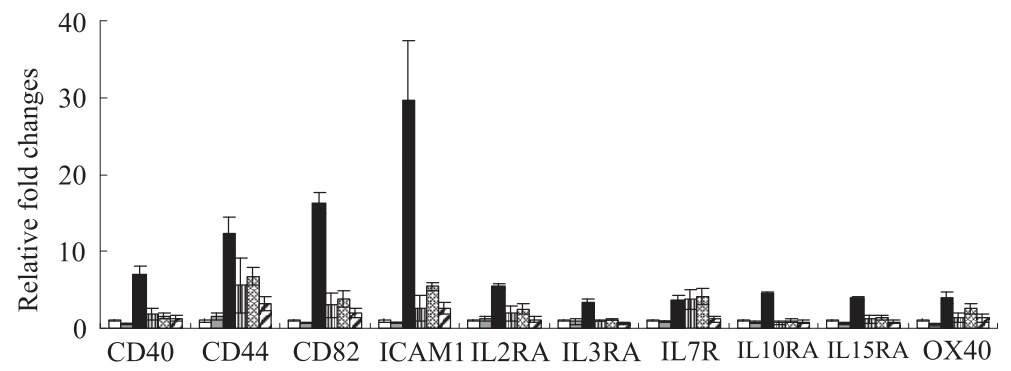

IFN-inducible genes

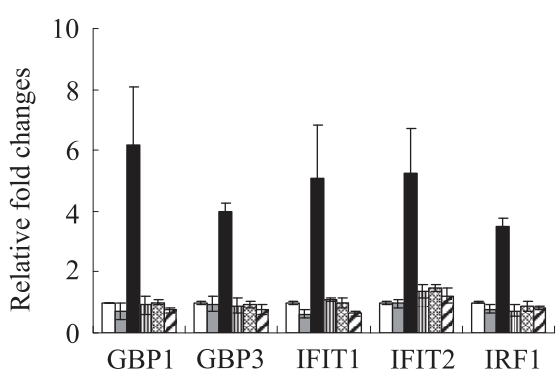

Others
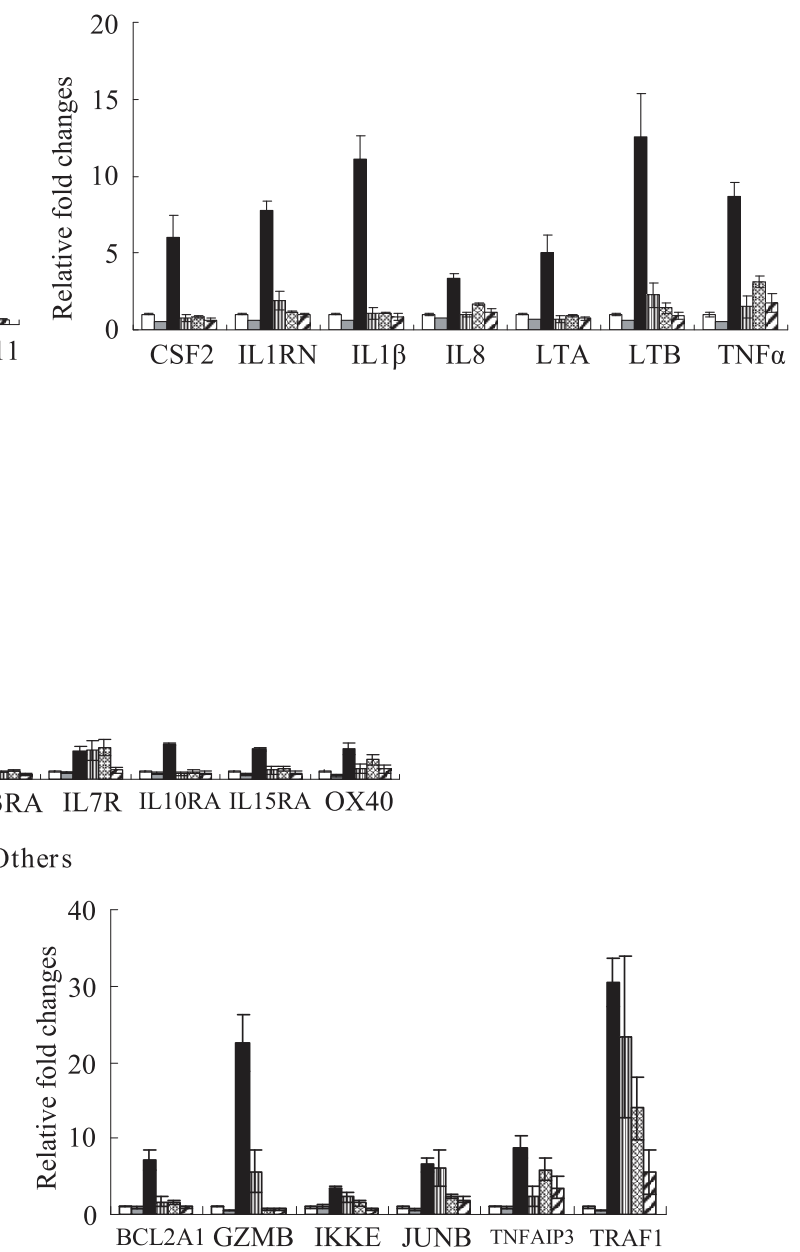

Fig. 3. The effect of fisetin on gene expression in $\mathrm{HMC}-1$ cells induced by activated $\mathrm{T}$ cell membranes

Cells were pre-incubated with or without the indicated concentration of fisetin for $15 \mathrm{~min}$. Resting (Tc-m) or phorbol-12-myristate 13-acetate (PMA)-activated T cell membranes (aTc-m) were then added and the cells incubated for a further $16 \mathrm{~h}$. Cellular gene expression was determined using a human DNA microarray (Genopal ARIH-GX, Mitsubishi Rayon). The degree of change (fold) was calculated compared to control cells. Data are expressed as mean \pm standard error from three different experiments.

$\square:$ HMC-1 alone, $\square: H M C-1+$ Tc-m,

:HMC-1+ -aTc-m+Fistin $25 \mu \mathrm{M}$,

:HMC-1+aTc-m, 四:HMC-1+ -aTc-m+Fistin $10 \mu \mathrm{M}$,

$\oslash:$ HMC-1+ -aTc-m+Fistin $50 \mu \mathrm{M}$.<smiles>O=c1c(O)c(-c2ccc(O)c(O)c2)oc2cc(O)ccc12</smiles>

Fig. 4. The chemical structure of fisetin cells $^{4}$. Our results suggest that $D$. rotundifolia and D. tokaiensis suppress activated $\mathrm{T}$ cell membrane-mediated mast cell activation in inflamed allergic tissues and at the site of parasitic infections.

\section{Evaluation of skin irritation on a reconstructed human epidermal model ${ }^{9}$}

The reconstructed human epidermal model has been accepted as a valid replacement of animal skin irritation testing for cosmetics, according to the European Center for the Validation of Alternative Methods $(\text { ECVAM })^{2}$. The model comprises cultured adult human 

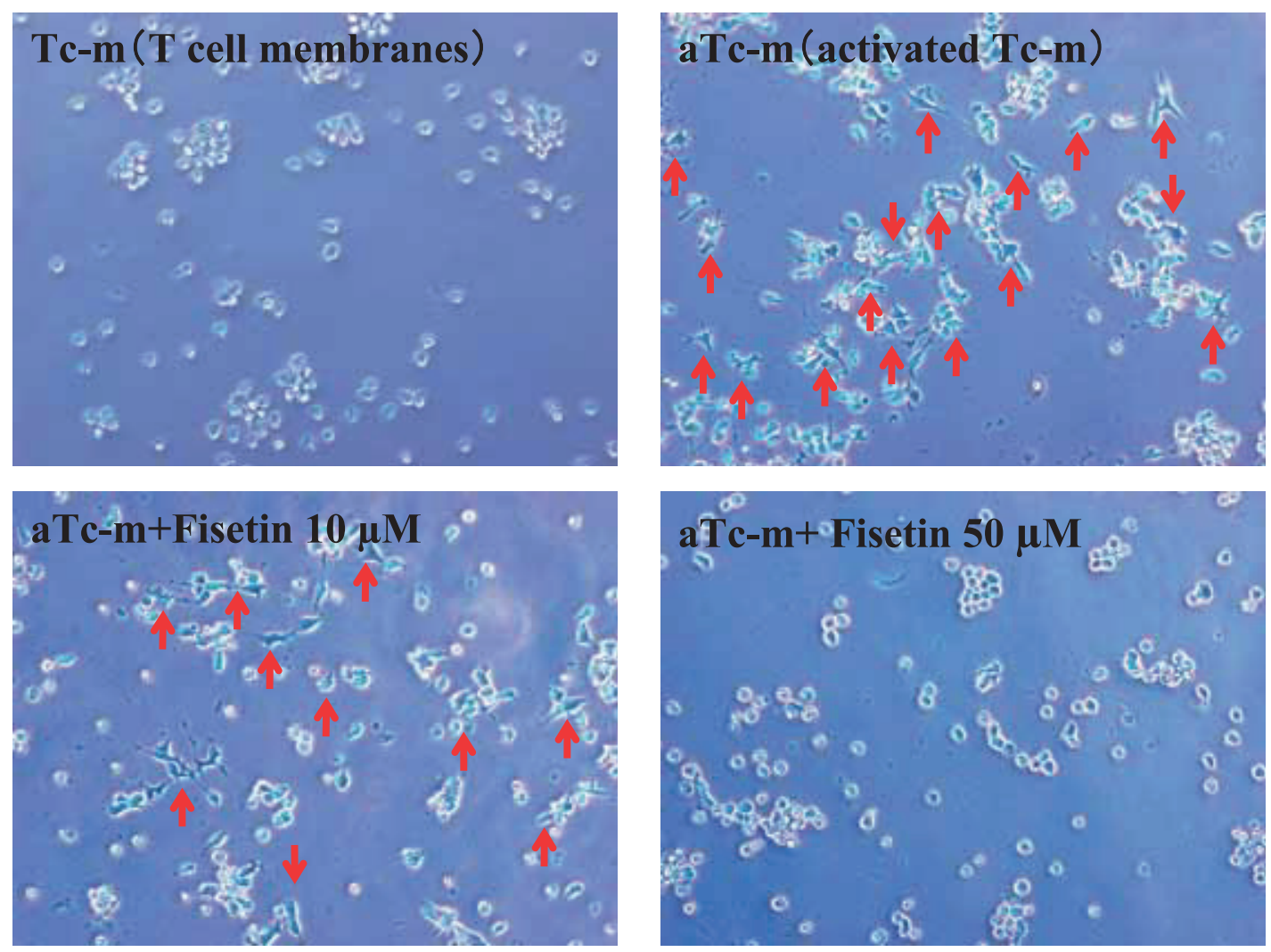

Fig. 5. Fisetin suppresses the morphological change in HMC-1 human mast cells induced by activated $\mathbf{T}$ cell membranes (aTc-m)

Cells were pre-incubated with or without the indicated concentration of fisetin for $15 \mathrm{~min}$. Resting (Tc-m) or PMA-activated T cell membranes (aTc-m) were then added and the cells incubated for $16 \mathrm{~h}$. HMC-1 cell morphology was observed by phase contrast microscopy.

The arrows indicate spreading cells.

keratinocytes on a collagen base, in conditions that permit their terminal differentiation and the reconstruction of an epidermis. To examine the sensitivity of the developed DNA microarray, we determined gene expression after the topical application of the mild skin irritant sodium dodecyl sulphate (SDS) to the reconstructed human epidermal model LabCyte ${ }^{\mathrm{TM}}$ EPI-MODEL (LabCyte), commercially available in Japan (Fig. 6). DNA microarray analysis indicated that expression of 10 of the 205 genes carried on the human DNA microarray was induced in a LabCyte culture by SDS irritation for $18 \mathrm{~h}$. We have shown for the first time that $0.075 \%$ SDS irritation significantly induced the expression of interleukin 1 receptor antagonist (IL-1RN), FOS-like antigen 1 (FOSL1), heat shock $70 \mathrm{kDa}$ protein 1A (HSPA $1)$, and myeloid differentiation primary response gene (88) (MYD88) in a LabCyte tissue. As IL1 $\alpha$ is quickly released in situations of epidermal injury, the ECVAMvalidated protocol recommends determining the amount of IL $1 \alpha$ protein in the culture medium to evaluate the skin irritation ${ }^{3}$. DNA microarray and RT-PCR analysis showed that $0.05 \%$ and/or $0.075 \%$ SDS induced the expression of IL1 $\alpha$. Enzyme-linked immunosorbent assay (ELISA) confirmed that $0.075 \%$ SDS induced the secretion of IL $1 \alpha$ from the LabCyte culture to the medium.

Thus, we evaluated the inflammatory responses in macrophages, mast cell activation associated with $\mathrm{T}$ cell-mediated allergic inflammatory response and skin irritation on a reconstructed human epidermal model using a DNA microarray carrying genes related to immunity, inflammation and housekeeping. Using the same methods, we found that bitter gourd exhibited an antiinflammatory effect, and fisetin and Drosera Herba possess a suppressive effect on mast cell activation by activated $\mathrm{T}$ cell membranes. The DNA microarray is useful for determining gene expression related to allergy, inflammation and irritation in human and mouse tissues. It can be a useful and efficient tool for evaluating the anti-allergy and anti-inflammatory effects of food components. 
$\mathrm{a}$

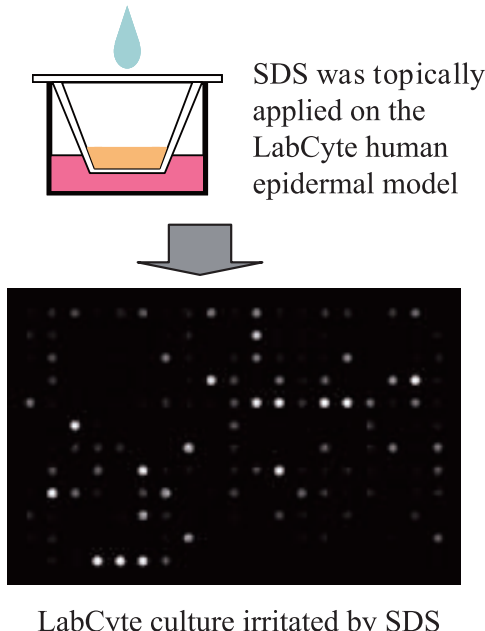

b

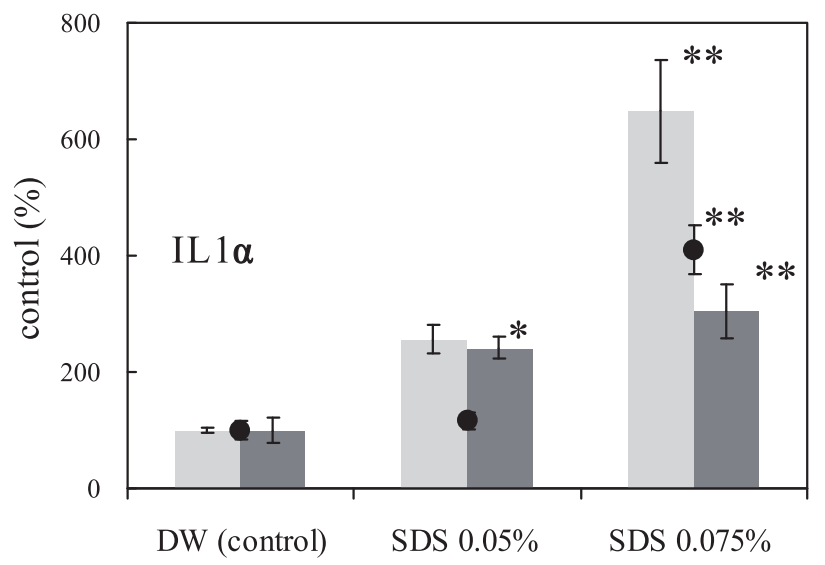

Fig. 6. Evaluation of sodium dodecyl sulphate (SDS)-induced skin irritation in a reconstructed human epidermal model, LabCyteTM EPI-MODEL

(a) : DNA microarray image of reconstructed human epidermal model stimulated by SDS

(b) : SDS induced a dose-dependent IL $1 \alpha$ release into the medium and mRNA expression in the LabCyte model. The cultures were treated with distilled water (DW) and $0.05,0.075 \%$ (w/w) SDS solution for $18 \mathrm{~h}$. The amount of released IL1 $\alpha$ was determined by ELISA. Gene expression levels of IL1 $\alpha$ determined by DNA microarray (Genopal ARIH-GX) and RT-PCR were normalized to GAPDH. Results were plotted relative to those in cultures treated with DW (control). All assays were performed in triplicate, and data are expressed as means \pm SE., $\mathrm{P}<$ $0.05 ;{ }^{* *}, \mathrm{P}<0.01$ (two-sided); significantly different from the group treated with DW by Bonferroni-type multiple test.

: RT-PCR, : DNA microarray, : ELISA.

\section{Acknowledgments}

This work was financially supported in part by grants from the Ministry of Agriculture, Forestry and Fisheries and by a grant-in-aid for Science Research (21500814). We wish to thank Professor J. H. Butterfield and Professor A. Kirshenbaum for supplying HMC-1 cells and LAD2 cells, respectively. We also thank Yokogawa Electric Co. for the loan of a DNA microarray reader and technical advice.

\section{References}

1. Arai, Y. et al. (2000) Dietary intakes of flavonols, flavones and isoflavones by Japanese women and the inverse correlation between quercetin intake and plasma LDL cholesterol concentration. J. Nutr., 130, 22432250.

2. ECVAM; Based on "Statement on the validity of in vitro tests for skin irritation." ESAC, ECVAM. April, 27 th, 2007.
3. ECVAM SIVS: Final draft performance standards version 2007-05-25.

4. Fukushima, K. et al. (2009) Drosera rotundifolia and Drosera tokaiensis suppress the activation of HMC-1 human mast cells. J. Ethnopharmacology, 125, 90-96.

5. Hirano, T. et al. (2004) Flavonoids such as luteolin, fisetin and apigenin are inhibitors of interleukin-4 and interleukin-13 production by activated human basophils. Int. Arch. Allergy Immunol., 134, 135-140.

6. Kawai, M. et al. (2007) Flavonoids and related compounds as anti-allergic substances. Allergol. Int., 56, $113-123$.

7. Kobori, M. et al. (2008) Bitter gourd suppresses lipopolysaccharide-induced inflammatory responses. $J$. Agric. Food Chem., 56, 4004-4011.

8. Nagai, K. et al. (2009) The hydroxyflavone, fisetin, suppresses mast cell activation induced by interaction with activated T cell membranes. Br. J. Pharmacol., 158, 907-919.

9. Niwa, M. et al. (2009) Evaluation of the skin irritation using a DNA microarray on a reconstructed human epidermal model. Biol. Pharm. Bull., 32, 203-208.

10. Park, H. et al. (2008) Flavonoids inhibit histamine release and expression of proinflammatory cytokines in mast cells. Arch. Pharm. Res., 31, 1303-1311. 